http://dx.doi.org/10.21611/qirt.1994.036

\title{
Numerical modelling of the defect response in pulsed video thermography on samples with finite optical penetration
}

by WALLE G. *, BURGSCHWEIGER G. ${ }^{\circ}$ and NETZELMANN U.*

* Fraunhofer-Institute for Nondestructive Testing, Saarbrücken, Germany

- Technical University "Otto von Guericke", Magdeburg, Germany

\begin{abstract} finite optical penetration depth in polymers. of about four times the optical penetration depth.

Nomenclature

T temperature

$t \quad$ time

$\lambda$ thermal conductivity

$\rho$ density

C specific heat capacity

$\gamma \quad$ optical absorption coefficient

$\Delta \quad$ Laplace operator

$z \quad$ coordinate normal to the surface

$I_{0}(t)$ time-dependence of heating pulse intensity $\left(\mathrm{W} / \mathrm{m}^{2}\right)$

$a_{v} \quad h e a t$ released per unit volume $\left(\mathrm{W} / \mathrm{m}^{3}\right)$
\end{abstract}

The results of one- and two-dimensional finite volume (FV) model calculations, based on the instationary heat diffusion theory, are applied to pulsed video thermography. A special regard is given to the optical absorption of light at the specimen surface and to the

The maximum temperature at surface is found to decrease significantly compared to opaque samples. The temperature contrast of a defect of finite size is affected to a depth

\section{Introduction}

In pulsed video thermography the instationary thermal diffusion process is usually initiated by flash tube illumination. Heat generation is caused by absorption of light in a thin layer at the material surface. If the material to be tested is a polymer or a ceramic, the optical penetration of the excitation light may be significant. In this case, the timedependent temperature response can be solved analytically for the one-dimensional heat flow and assuming Beer's absorption law [1, 2] or for subsurface absorption layers [3]. In short, deviations from the behavior of a surface absorber have to be expected for times $t$ where the optical penetration depth $1 / \gamma$ is still larger or comparable to the thermal diffusion distance $\sqrt{ }(\alpha t)$ ( $\alpha$ : thermal diffusivity). The maximum surface temperature is reduced for finite penetration.

\section{Numerical calculation}

\subsection{Method}

The situation is more complicated to analyze when the influence of the spatial/temporal response of a finite subsurface defect in an optically partially transparent 
http://dx.doi.org/10.21611/qirt.1994.036

material is of interest. We have set up a numerical algorithm using the finite volume method to solve the diffusion equation for rotational symmetry, assuming cylindrical samples with cylindrical subsurface defects. (Fig. 1).

The finite volume method is based on the rule of conservation of energy within each finite volume. The total volume of the sample is divided in a number of non-overlapping "finite volumes" and for the neighbored volumes the conditions for continuity of the heat flows are used. In the case of rotational symmetry cylindrical coordinates are used for the mathematical formulation of the finite volume method. The discretization is not uniform over the whole volume but a finer discretisation is selected for regions with higher temperature gradients, for instance, near the specimen surface [4].

The instationary heat diffusion is described by the equation:

$$
\rho c \frac{d T}{d t}-\lambda \cdot \Delta \mathrm{T}=\mathrm{q}_{\mathrm{v}} \text {. }
$$

In the case of heating by optical absorption one can write:

$$
q_{v}=I_{0}(t) \cdot \gamma e^{-\gamma z} \text {. }
$$

Different temporal profiles of the optical excitation can be considered: rectangular, gaussian or logarithmic gaussian shape heating pulse profiles.

In a first step one-dimensional calculations have been performed for polyvinyl-chloride (PVC). Experiments were performed on a pulse-video thermographic testing system described elsewhere [5], [6].

The numerical curves based on a gaussian distributed excitation pulse of $9 \mathbf{m s}$ width have been fitted to experimental data, resulting in a value of $\gamma=1.2 .10^{4} \mathrm{~m}^{-1}$ or an optical penetration depth of $80 \mu \mathrm{m}$.

As expected, the temperature response is higher and the maximum temperature is reached earlier for higher absorption coefficients (Fig. 2).

The value for $\gamma$ determined in the fit is taken as an input parameter for the twodimensional heat diffusion calculations of the temperature response of cylindrical subsurface defects in PVC.

\subsection{One-dimensional calculations for a layer characterization}

Using the optical penetration depth, calculated in 2.1 for PVC (80 $\mu \mathrm{m})$, onedimensional calculations were carried out for different PVC-layer thicknesses on a steel substrate. A homogenous pulsed heating with a gaussian pulse profile was assumed. The calculations are shown in figure 3 in comparison to results calculated with an optical absorption coefficient of $\gamma=10^{7} 1 / \mathrm{m}$ which means that light is absorbed in a layer very close to the surface.

The results show that there is a strong influence of the finite optical absorption depth on the maximum surface temperature and on the time dependence of temperature for all three layer thicknesses. Furthermore, one can find a significant effect of the layer thickness on the time dependence of the temperature at the specimen surface. With the help of such model calculations it should be possible to measure the layer thickness of layered materials by analyzing the cooling process at the specimen surface after pulsed heating.

\subsection{Two-dimensional calculations at specimen with artificial defects}

In figures 4 and 5 the results of two-dimensional FV-calculations are shown for a PVC sample with defects in different depths below the surface. The calculations were carried 
out on one hand having regard to the finite optical penetration depth and on the other hand assuming that the light is absorbed in a layer very close to the surface. The figures show that for a defect depth of $0.25 \mathrm{~mm}$ there is still a significant influence of the sptical penetration depth on the temperature contrast $\Delta T$ of that defect, whereas for a depth of $0.5 \mathrm{~mm}$ the influence is already weak.

So one can summarize that taking the effect of finite optical penetration into consideration, the absolute temperature at specimen surface is much lower than otherwise and the temperature contrasts of defects close to the surface (up to a depth of about four times the optical penetration depth) is also decreased in comparison to an absorption in a very thin surface layer.

Further calculations were carried out with defects with diameters between 0.5 and 4.0 $\mathrm{mm}$ in a depth of 0.25 to $4 \mathrm{~mm}$ below the surface. The calculations show a nearly linear dependence of the maximum temperature contrast $\left(\Delta T_{\text {max }}\right)$ of the defect diameter up to about $4 \mathrm{~mm}$ (Fig. 6). For larger diameters, especially for defects close to the surface, a saturation behavior can be recognized. In dependence of defect depth one can find a strong decrease in the temperature contrast (Fig. 7).

Calculations concerning the time-dependence of the temperature contrast were c:arried out. On the one hand, the time was calculated in which the temperature contrast $\Delta \mathrm{T}$

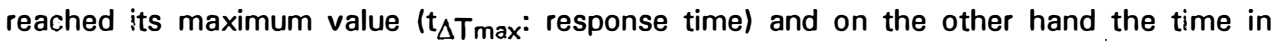
which the temperature reached $50 \%$ of the maximum temperature contrast $\Delta T_{\max }\left(t_{\Delta T 50}\right)$. The two characteristic times show a clear dependence on the defect depth (Fig. 8;. One can see that the response time and especially the time $t_{\Delta T 50}$ is much more dependent on the defect depth than on the defect diameter (Fig. 9).

\section{Conclusions}

The results of the one-dimensional finite volume calculations show that the finite optical penetration depth in materials such as polymers or ceramics can significantly influence the time dependence of the temperature at the specimen surface during and after heating. The maximum temperature at the surface is much lower for sinite optical penetration than for a optical absorption close to the surface.

Furthermore the results of two-dimensional calculations show that the temperature contrast of a defect is significantly affected by the finite optical penetration depth up to a defect depth of about four times of the optical penetration depth. The smaller the defect depth is, the more significant is the reduction of the temperature contrast in relation to the contrast calculated for a surface absorber.

\section{REFERENCES}

[1] IMHOF (R.E.), BIRCH (D.J.S.), THORNLEY (F.R.), GILCHRIST (J.R.), STRIVENS (T.A.) and J. Phys.E: Sci. Instr. 17, 521 (1984)

[2] LAU (S.K.) ALMOND (D.P.) and PATEL (P.M.) J. Appl. Phys. 24, 428 (1991)

[3] AAMODT (L.), SPICER (J.) and MURPHY (J.), Journal de Physique, to be published

[4] BURGSCHWEIGER (J.), Diploma Thesis, TU Magdeburg and FhG-IzfP Saarbrücken, 1993

[5] NETZELMANN (U.), WALLE (G.) DOBMANN (G.): "Real-time 3D representation of timeresolved infrared thermographic data", in: "Quantitative infrared thermography", BALAGEAS (D.) BUSSE (G.) and CARLOMAGNO (G.M.) (eds.), (Editions Europeenes Thermique et Industrie,Paris, 1992), p. 239-242.

[6] WALLE (G.) "Impuls-Video-Thermographie", Materialprüfung, Berlin, 1994, S. 86-88. 
http://dx.doi.org/10.21611/qirt.1994.036

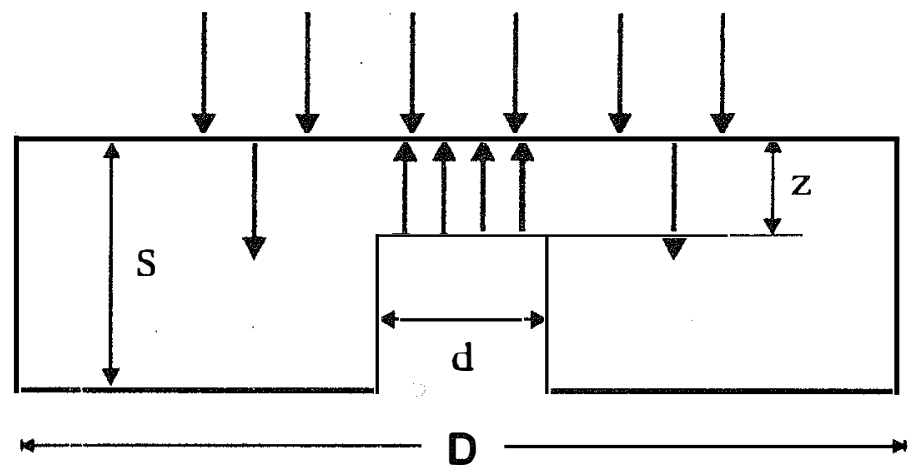

Fig.1 - Geometry of sample with defects

$\mathrm{T}[\mathrm{k}]$ surface temperature

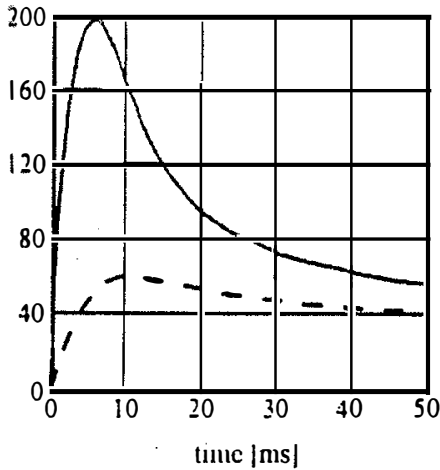

- PVC sample

- plate thickness: $1 \mathrm{~mm}$

- enery input: $20 \mathrm{~kJ} / \mathrm{m}^{2}$

- temporal gaussian heating pulse

$-\sigma_{\mathrm{t}}=9 \mathrm{~ms}$

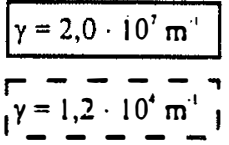

Fig.2 - Influence of $\gamma$ on cooling curve

$\mathrm{T}[\mathrm{K}] \quad$ Surface temperature

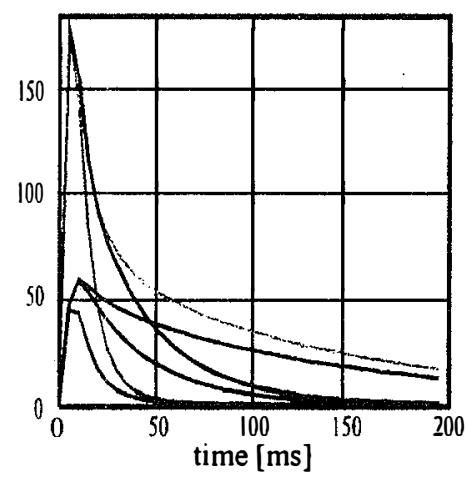

PVC layers on steel plate

(thickness $1 \mathrm{~mm}$ )

- energy input $20 \mathrm{~kJ} / \mathrm{m}^{2}$

- Gaussian distribution $\sigma_{\mathrm{t}}=9 \mathrm{~ms}$

layer thickness $[\mathrm{mm}] / \gamma\left[\mathrm{m}^{.1}\right]$

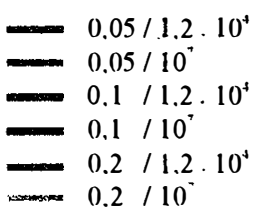

Fig.3 - Influence of $\gamma$ on cooling curve: different layer thicknesses 
http://dx.doi.org/10.21611/qirt.1994.036

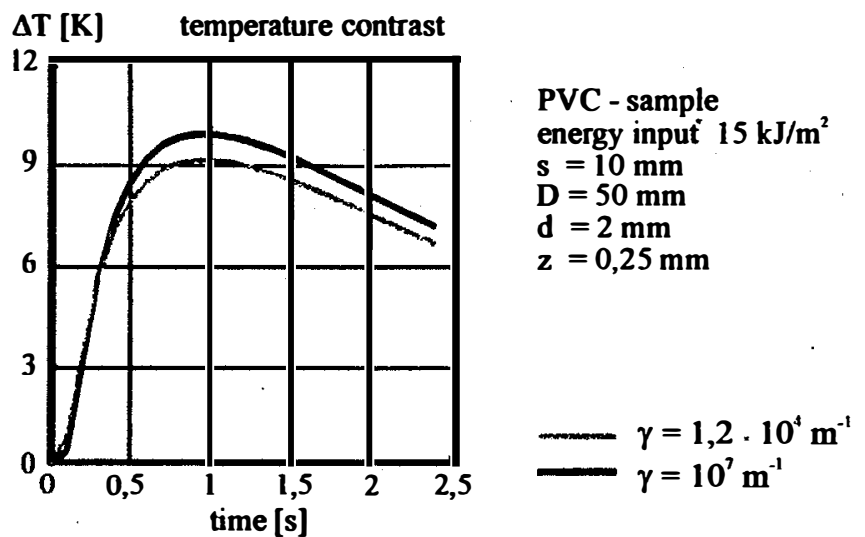

Fig.4 - Influence of the optical absomtion coefficient on defect contrast

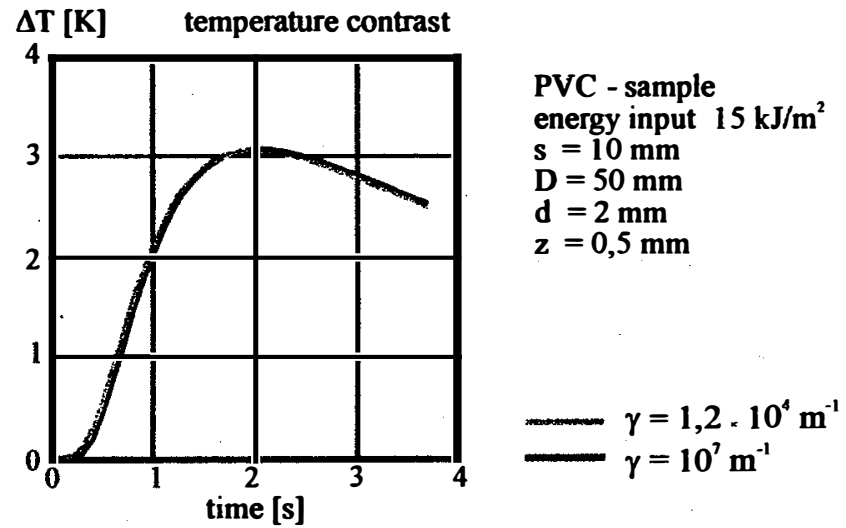

Fig.5 - Influence of the optical absorption coefficient on defect contrast

$\Delta T_{\operatorname{mx}}[K]$ temperature contrast

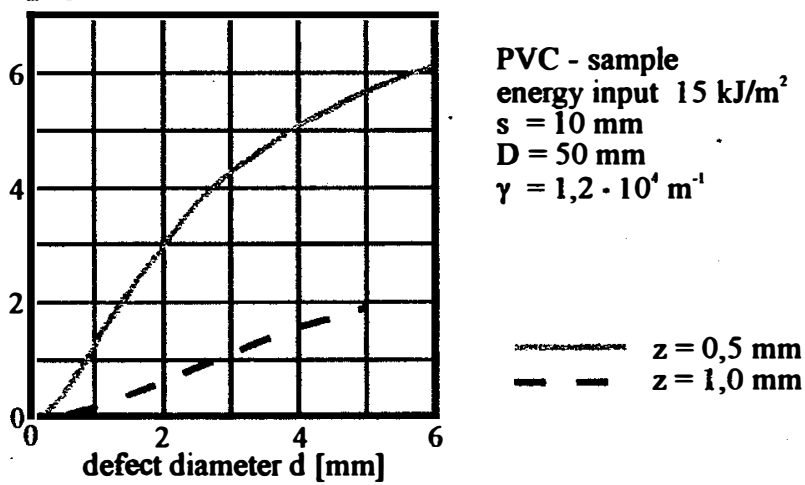

Fig. $6-\Delta T_{\max }$ as a function of defect diameter 
http://dx.doi.org/10.21611/qirt.1994.036

$\Delta T$ max $[K]$ temperature contrast

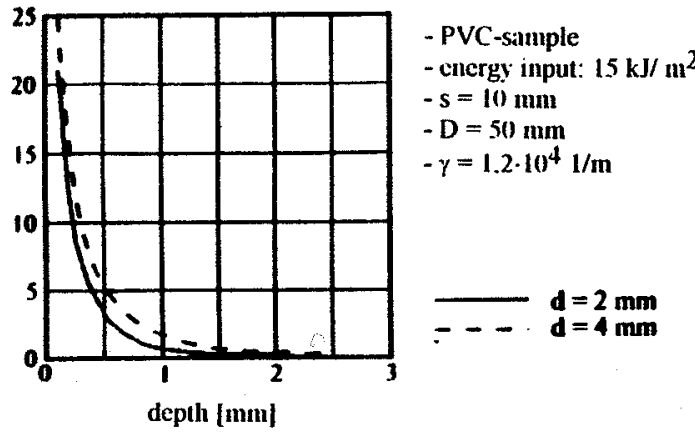

Fig.7 $-\Delta T_{\text {MAX }}$ as a function of defect depth

$t_{\text {aTmax }} / t_{x т s 0}[s]$ time of max.contrast / $50 \%$ of max. contrast

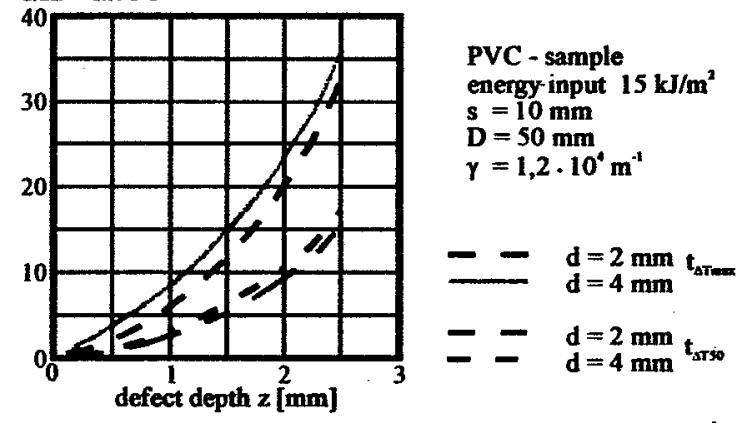

Fig.8 - Time of $\Delta T_{M A X}$ and of $50 \%$ of $\Delta T_{M A X}$ as a function of defect depth

$t_{\Delta T \max } / t_{\Delta T 50}[s]$ time of $\max$. contrast / $50 \%$ of max. contrast

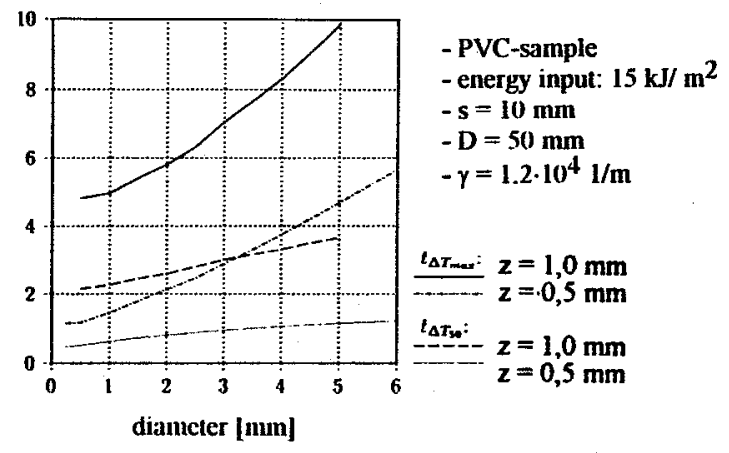

Fig.9 - Time $\Delta T_{\text {MAX }}$ as a function of defect diameter 